\title{
THE SURGERY OBSTRUCTION OF A DISJOINT UNION
}

\section{ANDREW RANICKI}

The surgery obstruction $\sigma_{*}(f, b) \in L_{n}\left(\pi_{1}(X)\right)$ of an $n$-dimensional degree 1 normal $\operatorname{map}(f, b): M \rightarrow X$ (in the sense of Browder [1] and Wall [7]) was formulated in [6] as the quadratic Poincare cobordism class of a pair $(C, \psi)$ consisting of an $n$-dimensional $\mathbb{Z}\left[\pi_{1}(X)\right]$-module chain complex $C$ and a chain level quadratic structure $\psi$ inducing Poincare duality $H^{n-{ }^{*}}(C) \neg H_{*}(C)$. If the manifold $M=\bigcup_{i=1}^{N} M_{i}$ is the disjoint union of manifolds $M_{i}$ it is natural to seek an expression for the surgery obstruction of $(f, b)$ in terms of quadratic structures defined by the restrictions $\left(f_{i}, b_{i}\right)=(f, b) \mid: M_{i} \rightarrow X$, which are normal maps of degree $d_{i}$ with

$$
f_{i}\left[M_{i}\right]=d_{i}[X] \in H_{n}(X), \quad d_{i} \in \mathbb{Z}, \quad \sum_{i=1}^{N} d_{i}=1 .
$$

The algebraic theory of surgery of [6] is here used to provide such an expression, describing the pair $(C, \psi)$ in terms of similar pairs $\left(C_{i}, \psi_{i}\right)$ which are associated to $\left(f_{i}, b_{i}\right)$. For the sake of simplicity we shall be working with the oriented case-the unoriented case is exactly the same, but with more complicated terminology.

I should like to thank Julius Shaneson and William Browder for conversations which stimulated my interest in this question.

As in [6] we shall actually be working with normal maps of geometric Poincaré complexes. Some care must be exercised about the precise definition of such normal maps (cf. Brumfiel and Milgram [4], for one possible definition).

A degree d normal map of $n$-dimensional geometric Poincaré complexes

$$
(f, b): M \rightarrow X
$$

is a map $f: M \rightarrow X$ such that $f_{*}[M]=d[X] \in H_{n}(X)(d \in \mathbb{Z})$, together with a map of $(k-1)$-spherical Spivak normal fibrations $b: v_{M} \rightarrow v_{X}$, and with preferred spherical generators $\rho_{M} \in \pi_{n+k}\left(T\left(v_{M}\right)\right), \rho_{X} \in \pi_{n+k}\left(T\left(v_{X}\right)\right)$. The latter are to be such that $h\left(\rho_{M}\right) \cap U_{v_{M}}=[M] \in H_{n}(M) \quad\left(h=\right.$ Hurewicz $\quad$ map: $\quad \pi_{n+k}\left(T\left(v_{M}\right)\right) \rightarrow \dot{H}_{n+k}\left(T\left(v_{M}\right)\right)$, $U_{v_{M}}=$ Thom class $\in H^{k}\left(T\left(v_{M}\right)\right), H=$ reduced (co)homology, $T\left(v_{M}\right)=$ Thom space), and similarly for $\rho_{X}$. In the case $d=1$ we no longer require $T(b)_{*}\left(\rho_{M}\right)=\rho_{X} \in \pi_{n+k}^{S}\left(T\left(v_{X}\right)\right)$, as we did in the definition of a degree 1 normal map in $[6]$.

A spherical generator $\rho_{X} \in \pi_{n+k}\left(T\left(v_{X}\right)\right)$ for the Thom space $T\left(v_{X}\right)$ of a $(k-1)$ spherical Spivak normal fibration $v_{X}: X \rightarrow B S G(k)$ of an $n$-dimensional geometric Poincaré complex $X$ determines an $S$-duality map

$$
\alpha_{X}: S^{n+k} \stackrel{\rho_{X}}{\rightarrow} T\left(v_{X}\right) \stackrel{\Delta}{\rightarrow} X_{+} \wedge T\left(v_{X}\right),
$$

Received 2 January. 1979; revised 10 February, 1979.

[J London Math. Soc. (2), 20 (1979), 559-566] 
with $X_{+}=X \cup\{p t$. $\}$ and $\Delta$ induced by the diagonal map. If $\tilde{X}$ is a covering of $X$ with group of covering translations $\pi$ then according to [6] there is defined also a $\pi$-equivariant $S$-duality ("S$\pi$-duality") map

$$
\alpha_{\bar{X}}: S^{n+k} \stackrel{\rho_{X}}{\rightarrow} T\left(v_{X}\right) \stackrel{\Delta}{\rightarrow} \tilde{X}_{+} \wedge_{\pi} T\left(v_{X}\right),
$$

with $v_{\tilde{X}}: \tilde{X} \rightarrow X \stackrel{v_{x}}{\rightarrow} B S G(k)$ and $\tilde{X}_{+} \wedge_{\pi} T\left(v_{\tilde{X}}\right)$ the quotient of $\tilde{X}_{+} \wedge T\left(v_{\tilde{X}}\right)$ by the diagonal $\pi$-action.

The chain Umkehr of an $n$-dimensional degree $d$ normal map $(f, b): M \rightarrow X$ is the composite $\mathbb{Z}\left[\pi_{1}(X)\right]$-module chain map (defined up to chain homotopy)

$$
f^{\prime}: C(\tilde{X}) \stackrel{([X] \cap-)^{-1}}{\longrightarrow} C(\tilde{X})^{n-*]^{*}} \longrightarrow C(\tilde{M})^{n-*[M] \cap-} \longrightarrow(\tilde{M}),
$$

with $\tilde{X}$ the universal cover of $X, \tilde{M}$ the cover of $M$ induced from $\tilde{X}$ by $f$, and

$$
C(\tilde{X})^{n-*}=\operatorname{Hom}_{\mathbb{Z}\left[\pi_{1}(X)\right]}\left(C(\tilde{X})_{n-*}, \mathbb{Z}\left[\pi_{1}(X)\right]\right) .
$$

The chain Umkehr is such that there is defined a chain homotopy commutative diagram

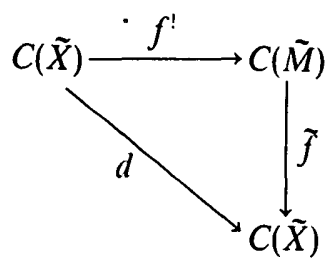

The homotopy Umkehr of $(f, b)$ is the stable $\pi_{1}(X)$-equivariant homotopy class of stable $\pi_{1}(X)$-equivariant maps $F: \Sigma^{\infty} \tilde{X}_{+} \rightarrow \Sigma^{\infty} \tilde{M}_{+} S \pi_{1}(X)$-dual to the induced map of Thom spaces $T(\tilde{b}): T\left(v_{\tilde{M}}\right) \rightarrow T\left(v_{\tilde{X}}\right)$, using the $S \pi_{1}(X)$-duality maps $\alpha_{\bar{M}}, \alpha_{\tilde{X}}$ determined by $\rho_{M}, \rho_{X}$. The homotopy Umkehr $F$ induces the chain Umkehr $f^{\prime}$ on the chain level. The homotopy degree of $(f, b)$ is the stable cohomotopy class $\delta \in\left[X_{+}, Q S^{0}\right]=\pi_{S}^{0}\left(X_{+}\right)$ $S$-dual to $T(b)_{*}\left(\rho_{M}\right) \in \pi_{n+k}^{S}\left(T\left(v_{X}\right)\right)$ under the $S$-duality isomorphism $\alpha_{X}: \pi_{n+k}^{S}\left(T\left(v_{X}\right)\right) \simeq \pi_{S}^{0}\left(X_{+}\right)$determined by $\rho_{X}$. The homotopy degree $\delta: X_{+} \rightarrow Q S^{0}$ sends $X$ to the component $Q_{d} S^{0}$ of $d \in H_{0}\left(Q S^{0}\right)=\mathbb{Z}$ in $Q S^{0}=\lim _{\vec{m}} \Omega^{m} S^{m}$. The homotopy Umkehr and the homotopy degree are related by a stable $\pi_{1}(X)$-equivariant homotopy commutative diagram

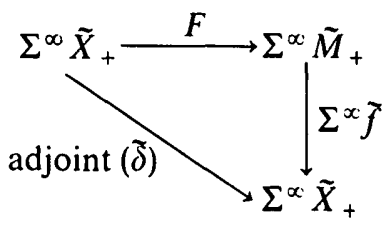

Inducing the previous diagram on the chain level, with $\tilde{\delta}: \tilde{X}_{+} \rightarrow X_{+} \stackrel{\delta}{\rightarrow} Q S^{0}$.

Given a group $\pi$, spaces with $\pi$-action $X, Y$ and a stable $\pi$-equivariant map 
$F: \Sigma^{\infty} X_{+} \rightarrow \Sigma^{\infty} Y_{+}$define the composite stable $\pi$-equivalent map

$$
X_{+} \stackrel{\text { adjoint }(F)}{\longrightarrow} \Omega^{\infty} \Sigma^{\infty} Y_{+} \stackrel{\text { stable homotopy projection }}{\longrightarrow}\left(E \mathbb{Z}_{2}\right)_{+} \wedge{Z_{2}}_{2}\left(Y_{+} \wedge Y_{+}\right)
$$

As in [6] call the induced abelian group morphisms

$$
\psi_{F}: H_{n}(X / \pi) \rightarrow Q_{n}(C(Y))=H_{n}\left(W \otimes_{\mathbb{Z}\left[\mathbb{Z}_{2}\right]}\left(C(Y) \otimes_{\mathbb{Z}[\pi]} C(Y)\right)\right)
$$

the quadratic construction. Here, the generator $T \in \mathbb{Z}_{2}$ acts on $Y_{+} \wedge Y_{+}=(Y \times Y)_{+}$by the transposition $(a, b) \mapsto(b, a)$, on $C(Y) \otimes_{\mathbb{Z}[\pi]} C(Y)$ by the signed transposition $a \otimes b \mapsto(-)^{|a| b \mid} b \otimes a$, and $W=C\left(E \mathbb{Z}_{2}\right)$ is the free $\mathbb{Z}\left[\mathbb{Z}_{2}\right]$-module resolution of $\mathbb{Z}$

$$
W: \ldots \rightarrow \mathbb{Z}\left[\mathbb{Z}_{2}\right] \stackrel{1+T}{\longrightarrow} \mathbb{Z}\left[\mathbb{Z}_{2}\right] \stackrel{1-T}{\longrightarrow} \mathbb{Z}\left[\mathbb{Z}_{2}\right] \rightarrow 0 .
$$

The quadratic construction $\psi_{F}$ depends only on the stable $\pi$-equivariant homotopyclass of $F$.

The quadratic signature of an $n$-dimensional degree 1 normal map $(f, b): M \rightarrow X$ is the quadratic Poincaré cobordism class

$$
\sigma_{*}(f, b)=\left(C\left(f^{\prime}\right), e_{\%} \psi_{F}[X] \in Q_{n}\left(C\left(f^{\prime}\right)\right)\right) \in L_{n}\left(\pi_{1}(X)\right)
$$

with $C\left(f^{\prime}\right)$ the algebraic mapping cone of the chain Umkehr $f^{\prime}: C(\tilde{X}) \rightarrow C(\tilde{M})$, $e: C(\tilde{M}) \rightarrow C\left(f^{\prime}\right)$ the projection, and $\psi_{F}[X] \in Q_{n}(C(\tilde{M}))$ the evaluation on the fundamental class $[X] \in H_{n}(X)$ of the quadratic construction $\psi_{F}$ on the homotopy Umkehr $F: \Sigma^{\infty} \tilde{X}_{+} \rightarrow \Sigma^{\infty} \tilde{M}_{+}$. We are not repeating here the many other definitions and constructions of [6] which might make this meaningful.

A degree 1 normal map $(f, b): M \rightarrow X$ in the sense of [1] and [7] determines a degree 1 normal map $(f, J b): M \rightarrow X$ in the present sense, with homotopy degree $1 \in \pi_{S}^{0}\left(X_{+}\right)$, as follows. Since $M$ is now a manifold we can take for $\left(v_{M}: M \rightarrow B S G(k), \rho_{M}: S^{n+k} \rightarrow T\left(\rho_{M}\right)\right)$ the Spivak normal structure given by an embedding of $M$ in $S^{n+k}(k \gg n)$ as a manifold, not just as a Poincaré complex, and we can define $\rho_{X}=T(b)_{*}\left(\rho_{M}\right) \in \pi_{n+k}\left(T\left(v_{X}\right)\right)$. According to [6] the surgery obstruction of $(f, b)$ is the quadratic signature $\sigma_{*}(f, J b) \in L_{n}\left(\pi_{1}(X)\right)$.

Our description of the quadratic signature $\sigma_{*}(f, b) \in L_{n}\left(\pi_{1}(X)\right)$ of a disjoint union degree 1 normal map $(f, b)=\bigcup_{i}\left(f_{i}, b_{i}\right): \bigcup_{i} M_{i} \rightarrow X$ is based on the following quadratic property of the quadratic construction $\psi$, which is an easy consequence of its construction.

LEMMA. Given a group $\pi$, spaces with $\pi$-action $X, Y_{i}$ and stable $\pi$-equivariant maps $F_{i}: \Sigma^{\infty} X_{+} \rightarrow \Sigma^{\infty}\left(Y_{i}\right)_{+}(1 \leqslant i \leqslant N)$ track addition defines a stable $\pi$-equivariant map

$$
F=\bigvee_{i} F_{i}: \Sigma^{\infty} X_{+} \rightarrow \Sigma^{\infty}\left(\bigcup_{i=1}^{N} Y_{i}\right)_{+}=\bigvee_{i=1}^{N} \Sigma^{\infty}\left(Y_{i}\right)_{+}
$$


The quadratic construction on $F$ is given by

$$
\begin{aligned}
\psi_{F} & =\left(\begin{array}{c}
\bigoplus_{i} \psi_{F_{i}} \\
\bigoplus_{i<j}-\left(f_{i} \otimes f_{j}\right) \Delta
\end{array}\right): H_{n}(X / \pi) \rightarrow Q_{n}\left(C\left(\bigcup_{i=1}^{N} Y_{i}\right)\right) \\
& =\bigoplus_{i=1}^{N} Q_{n}\left(C\left(Y_{i}\right)\right) \oplus \bigoplus_{i<j} H_{n}\left(C\left(Y_{i}\right) \otimes_{\mathbb{Z}[\pi]} C\left(Y_{j}\right)\right),
\end{aligned}
$$

with $f_{i}: C(X) \rightarrow C\left(Y_{i}\right)$ the $\mathbb{Z}[\pi]$-module chain map induced by $F_{i}$, and

$$
\Delta: H_{n}(X / \pi) \rightarrow H_{n}\left(C(X) \otimes_{\mathbb{Z}[\pi]} C(X)\right)
$$

the map induced by a $\pi$-equivariant diagonal chain approximation $\Delta: C(X) \rightarrow C(X) \otimes_{\mathbb{Z}} C(X)$.

The disjoint union of $n$-dimensional degree $d_{i}$ normal maps $\left(f_{i}, b_{i}\right): M_{i} \rightarrow X$ $(1 \leqslant i \leqslant N)$ with the same Spivak normal structure $\left(v_{X}: X \rightarrow B S G(k), \rho_{X}: S^{n+k} \rightarrow T\left(v_{X}\right)\right)$ for $X$ is an $n$-dimensional degree $d=\sum_{i=1}^{N} d_{i}$ normal map

$$
(f, b)=\bigcup_{i=1}^{N}\left(f_{i}, b_{i}\right): \bigcup_{i=1}^{N} M_{i} \rightarrow X
$$

with chain Umkehr

$$
f^{\prime}=\bigoplus_{i} f_{i}^{\prime}: C(\tilde{X}) \rightarrow C\left(\bigcup_{i=1}^{N} \tilde{M}_{i}\right)=\bigoplus_{i=1}^{N} C\left(\tilde{M}_{i}\right)
$$

homotopy Umkehr

$$
F=\bigvee_{i} F_{i}: \Sigma^{\infty} \tilde{X}_{+} \rightarrow \Sigma^{\infty}\left(\bigcup_{i=1}^{N} \tilde{M}_{i}\right)_{+}=\bigvee_{i=1}^{N} \Sigma^{\infty}\left(\tilde{M}_{i}\right)_{+}
$$

and homotopy degree $\delta=\sum_{i=1}^{N} \delta_{i} \in \pi_{S}^{0}\left(X_{+}\right)$. Applying the lemma we have:

Proposition 1. The quadratic signature of an n-dimensional degree 1 normal map $(f, b)=\bigcup_{i=1}^{N}\left(f_{i}, b_{i}\right): \bigcup_{i=1}^{N} M_{i} \rightarrow X$ which is the disjoint union of degree $d_{i}$ normal maps $\left(f_{i}, b_{i}\right): M_{i} \rightarrow X\left(\sum_{i=1}^{N} d_{i}=1\right)$ is the quadratic Poincaré cobordism class

$$
\sigma_{*}(f, b)=\left(C\left(f^{\prime}\right), e_{\%} \psi_{F}[X] \in Q_{n}\left(C\left(f^{\prime}\right)\right)\right) \in L_{n}\left(\pi_{1}(X)\right),
$$


with $e: \bigoplus_{i=1}^{N} C\left(\tilde{M}_{i}\right) \rightarrow C\left(f^{\prime}\right)$ the projection and

$$
\begin{aligned}
\psi_{F}[X] & =\left(\bigoplus_{i=1}^{N} \psi_{f_{i}}[X], \bigoplus_{i<j}-\left(f_{i}^{!} \otimes f_{j}^{\prime}\right) \Delta[X]\right) \in Q_{n}\left(C\left(\bigcup_{i=1}^{N} \tilde{M}_{i}\right)\right) \\
& =\bigoplus_{i=1}^{N} Q_{n}\left(C\left(\tilde{M}_{i}\right)\right) \oplus \bigoplus_{i<j} H_{n}\left(C\left(\tilde{M}_{i}\right) \otimes_{\mathbb{Z}\left[\pi_{1}(X)\right]} C\left(\tilde{M}_{j}\right)\right) .
\end{aligned}
$$

One case is of particular interest: given a degree 0 normal map $(f, b): M \rightarrow X$ there is defined a degree 1 normal map

$$
(g, c)=(f \cup 1, b \cup 1): M \cup X \rightarrow X
$$

for which we can identify

$$
\begin{aligned}
g^{\prime} & =\left(\begin{array}{c}
f^{\prime} \\
1
\end{array}\right): C(\tilde{X}) \rightarrow C(\tilde{M} \cup \tilde{X})=C(\tilde{M}) \oplus C(\tilde{X}) \\
e & =\left(1 \quad-f^{\prime}\right): C(\tilde{M} \cup \tilde{X})=C(\tilde{M}) \oplus C(\tilde{X}) \rightarrow C\left(g^{\prime}\right)=C(\tilde{M}) \\
G & =F \vee 1: \Sigma^{\infty} \tilde{X}_{+} \rightarrow \Sigma^{\infty}(\tilde{M} \cup \tilde{X})_{+}=\Sigma^{\infty} \tilde{M}_{+} \vee \Sigma^{\infty} \tilde{X}_{+} .
\end{aligned}
$$

Substituting this in the expression of Proposition 1 we obtain:

Proposition 2. The quadratic signature of the degree 1 normal map $(g, c)=(f \cup 1, b \cup 1)$ is the quadratic Poincaré cobordism class

$$
\sigma_{*}(g, c)=\left(C(\tilde{M}), \quad \psi_{F}[X]+\left(f^{!} \otimes f^{\prime}\right) \Delta[X] \in Q_{n}(C(\tilde{M}))\right) \in L_{n}\left(\pi_{1}(X)\right),
$$

where $\left(f^{\prime} \otimes f^{\prime}\right) \Delta[X] \in Q_{n}(C(\tilde{M}))$ is the image of

$$
\left(f^{!} \otimes f^{\prime}\right) \Delta[X] \in H_{n}\left(C(\tilde{M}) \otimes_{\mathbb{Z}\left[\pi_{1}(X)\right]} C(\tilde{M})\right)
$$

under the abelian group morphism

$$
\begin{gathered}
H_{n}\left(C(\tilde{M}) \otimes_{\mathbb{Z}\left[\pi_{1}(X)\right]} C(\tilde{M})\right) \rightarrow Q_{n}(C(\tilde{M})) ; \\
\phi \mapsto \psi, \quad \psi_{s}= \begin{cases}\phi \in\left(C(\tilde{M}) \otimes_{\mathbb{Z}\left[\pi_{1}(X)\right]} C(\tilde{M})\right)_{n} & s=0 \\
0 \in\left(C(\tilde{M}) \otimes_{\mathbb{Z}\left[\pi_{1}(X)\right]} C(\tilde{M})\right)_{n-s} & s \geqslant 1 .\end{cases}
\end{gathered}
$$

In [6] there were also considered the symmetric $L$-groups $L^{n}(\pi)(n \geqslant 0)$ of a group $\pi$, and the symmetric signature invariant $\sigma^{*}(X) \in L^{n}\left(\pi_{1}(X)\right)$ of an $n$-dimensional geometric Poincaré complex $X$, both of which were originally introduced by Mishchenko. There are defined symmetrization maps

$$
1+T: L_{n}(\pi) \rightarrow L^{n}(\pi) \quad(n \geqslant 0)
$$


which are isomorphisms modulo 8-torsion. The quadratic signature $\sigma_{*}(f, b) \in L_{n}\left(\pi_{1}(X)\right)$ of a degree 1 normal map of $n$-dimensional geometric Poincaré complexes $(f, b): M \rightarrow X$ has symmetrization

$$
(1+T) \sigma_{*}(f, b)=\sigma^{*}(M)-\sigma^{*}(X) \in L^{n}\left(\pi_{1}(X)\right),
$$

where $\sigma^{*}(M) \in L^{n}\left(\pi_{1}(X)\right)$ is the image of $\sigma^{*}(M) \in L^{n}\left(\pi_{1}(M)\right)$ under the morphism induced by $f_{*}: \pi_{1}(M) \rightarrow \pi_{1}(X)$. If $X$ and $Y$ are $n$-dimensional geometric Poincaré complexes and there are given group morphisms $\pi_{1}(X) \rightarrow \pi, \pi_{1}(Y) \rightarrow \pi$ to the same group $\pi$ then

$$
\sigma^{*}(X \cup Y)=\sigma^{*}(X)+\sigma^{*}(Y) \in L^{n}(\pi) .
$$

(The symmetric signature is defined for disconnected geometric Poincare complexes using fundamental groupoids, exactly as in Wall [7].) Given a degree 1 normal map of . $n$-dimensional geometric Poincaré complexes which is a disjoint union

$$
(f, b)=\bigcup_{i=1}^{N}\left(f_{i}, b_{i}\right): \bigcup_{i=1}^{N} M_{i} \rightarrow X
$$

we thus have

$$
(1+T) \sigma_{*}(f, b)=\sum_{i=1}^{N} \sigma^{*}\left(M_{i}\right)-\sigma^{*}(X) \in L^{n}\left(\pi_{1}(X)\right)
$$

The semicharacteristic classes of Lee [5] are the images of the symmetric signature in appropriate Grothendieck groups of orthogonal representations, so that the semicharacteristic part of the surgery obstruction is additive on disjoint unions. I am grateful to C. T. C. Wall for drawing my attention to the relevance of [5].

For readers unfamiliar with the algebraic theory of surgery of [6] we shall express the simply-connected even-dimensional case of Proposition 2 in the language of Browder [1], using functional Steenrod squares. Indeed, this case has essentially already been worked out in $\$ 4$ of Browder [2].

Proposition 3. Let $(f, b): M \rightarrow X$ be a degree 0 normal map of 2i-dimensional geometric Poincare complexes. The image of the quadratic signature $\sigma_{*}(f \cup 1, b \cup 1) \in L_{2 i}\left(\pi_{1}(X)\right)$ of the degree 1 normal map $(f \cup 1, b \cup 1): M \cup X \rightarrow X$ in

$$
L_{2 i}(1)= \begin{cases}\mathbb{Z} & (i \equiv 0(\bmod 2)) \\ \mathbb{Z}_{2} & (i \equiv 1(\bmod 2))\end{cases}
$$

is just $\left\{\begin{array}{l}\frac{1}{8}(\text { the signature }) \\ \text { the Arf invariant }\end{array}\right.$ of the non-singular $(-)^{i}$ quadratic form $(G, \lambda, \mu)$ over the ring $A$ defined by

$$
G=\left\{\begin{array}{l}
H^{i}(M ; \mathbb{Z}) / \text { torsion } \\
H^{i}\left(M ; \mathbb{Z}_{2}\right)
\end{array}, \quad A=\left\{\begin{array}{l}
\mathbb{Z} \\
\mathbb{Z}_{2}
\end{array}\right.\right.
$$


$\lambda: G \times G \rightarrow A ; \quad(x, y) \mapsto\left\langle f^{\prime *}(x \cup y)+\left(f^{\prime *} x \cup f^{\prime *} y\right),[X]\right\rangle$

$$
=\langle x \cup y,[M]\rangle+\left\langle f^{! *} x \cup f^{\prime *} y,[X]\right\rangle
$$

$$
\mu: G \rightarrow A /\left\{a-(-)^{i} a \mid a \in A\right\}(=A) ; \quad z \mapsto\left\{\begin{array}{l}
\frac{1}{2} \lambda(z, z) \\
\left\langle S q_{h}^{i+1}\left(\Sigma^{k} l\right), \Sigma^{k}[X]\right\rangle+\langle z \cup z,[M]\rangle
\end{array}\right.
$$$$
\left(h=\left(\Sigma^{k} z\right) F-\Sigma^{k}\left(f^{! *} z\right) \in\left[\Sigma^{k} X_{+}, \Sigma^{k} K\left(\mathbb{Z}_{2}, i\right)\right], \quad z \in H^{i}\left(M ; \mathbb{Z}_{2}\right)=\left[M_{+}, K\left(\mathbb{Z}_{2}, i\right)\right],\right.
$$

$\iota=$ generator $\in H^{i}\left(K\left(\mathbb{Z}_{2}, i\right) ; \mathbb{Z}_{2}\right)=\mathbb{Z}_{2}, F: \Sigma^{k} X_{+} \rightarrow \Sigma^{k} M_{+}$( $k$ large $)$ is the $S$-dual of the induced map of Thom spaces $\left.T(b): T\left(v_{M}\right) \rightarrow T\left(v_{X}\right)\right)$.

(Of course, in Proposition 3-and below-we are really only using $A$-coefficient Poincaré duality, and not the universal $\mathbb{Z}\left[\pi_{1}(X)\right]$-coefficient Poincaré duality.

A finite $d$-sheeted covering $p: \bar{X} \rightarrow X$ of an $n$-dimensional geometric Poincaré complex $X$ determines a degree $d$ normal map $(p, b): \bar{X} \rightarrow X$ with homotopy degree the composite $X \stackrel{p}{\rightarrow} B \Sigma_{d} \rightarrow Q S^{0}$ of the classifying map $p: X \rightarrow B \Sigma_{d}$ and the canonical $\operatorname{map} B \Sigma_{d} \rightarrow Q_{d} S^{0} \hookrightarrow Q S^{0}$, as follows. Let $W$ be a closed regular neighbourhood of $X$ in $S^{n+k}$ for some embedding $X \leftrightarrow S^{n+k}(k>n+1)$, defining a Spivak normal structure $\left(v_{X}: X \rightarrow B S G(k), \rho_{X}: S^{n+k} \rightarrow T\left(v_{X}\right)\right)$ for $X$ by

$$
S^{k-1} \rightarrow \partial W \stackrel{v_{X}}{\rightarrow} W \simeq X, \quad \rho_{X}: S^{n+k} \stackrel{\text { collapse }}{\longrightarrow} S^{n+k} / \overline{S^{n+k}-W}=W / \partial W=T\left(v_{X}\right) .
$$

The induced cover $\bar{W}$ of $W$ has a trivialized tangent bundle, namely the pullback of the tangent bundle of $W$. This trivialization determines a regular homotopy class of immersions of $\bar{W}$ in $S^{n+k}$. Now $k>n+1$ and $\bar{W}$ has an $n$-dimensional spine, to wit $\bar{X}$, so that this class actually contains an embedding of $\bar{W}$ in $S^{n+k}$. Thus $\bar{W}$ is a closed regular neighbourhood of $\bar{X}$ for an embedding $\bar{X} \hookrightarrow S^{n+k}$, defining a Spivak normal structure $\left(v_{\bar{X}}: \bar{X} \rightarrow B S G(k), \rho_{\bar{X}}: S^{n+k} \rightarrow T\left(v_{\bar{X}}\right)\right)$. (For this line of argument I am indebted to Larry Taylor.) The quadratic signature

$$
\sigma_{*}(p \cup \cup 1, b \cup \cup 1) \in L_{n}\left(\pi_{1}(X)\right)
$$

of the degree 1 normal map

$$
(p \cup \cup 1, b \cup \cup 1): \bar{X} \cup \bigcup_{i=2}^{d}-X \rightarrow X
$$

is expressed by Proposition 1 in terms of the homotopy Umkehr $P: \Sigma^{\infty} \tilde{X}_{+} \rightarrow \Sigma^{\infty} \tilde{\bar{X}}_{+}$ $\left(=\bigvee_{d} \Sigma^{\infty} \tilde{X}_{+}\right)$, where $-X$ denotes $X$ with the opposite orientation. Note that the chain Umkehr $p^{\prime}: C(\tilde{X}) \rightarrow C(\tilde{\bar{X}})$ is just the usual chain level transfer of the cover $\tilde{p}: \tilde{\bar{X}} \rightarrow \tilde{X}$ of the universal cover $\tilde{X}$ of $X$ induced from $p: \bar{X} \rightarrow X$ by $\tilde{X} \rightarrow X$. In particular, for a double cover $(d=2)$ Proposition 2 gives a quadratic Poincaré cobordism class

$$
\sigma_{*}(p \cup \cup 1, b \cup \cup 1)=\left(C(\tilde{\bar{X}}), \psi_{p}[X]+\left(p^{\prime} \otimes p^{\prime}\right) \Delta[X]\right) \in L_{n}\left(\pi_{1}(X)\right) .
$$


A double cover $p: \bar{X} \rightarrow X$ determines yet another quadratic Poincaré cobordism class

$$
\sigma_{*}(X, p)=\left(C(\tilde{\bar{X}}), \psi_{P}[X]\right) \in L_{n}\left(\pi_{1}(X)\right) .
$$

If $n=2 i$ the image of $\sigma_{*}(X, p)$ in $L_{2 i}(1)$ is just

$$
\begin{cases}\frac{1}{8}(\text { the signature }) & (i \equiv 0(\bmod 2)) \\ \text { the Arf invariant } & (i \equiv 1(\bmod 2))\end{cases}
$$

of the non-singular $(-)^{i}$-quadratic form $(G, \lambda, \mu)$ over $A$ defined by

$$
G=\left\{\begin{array}{l}
H^{i}(\bar{X} ; \mathbb{Z}) / \text { torsion } \\
H^{i}\left(\bar{X} ; \mathbb{Z}_{2}\right)
\end{array}, A=\left\{\begin{array}{l}
\mathbb{Z} \\
\mathbb{Z}_{2}
\end{array}\right.\right.
$$

$\lambda: G \times G \rightarrow A ; \quad(x, y) \mapsto\left\langle p^{i *}(x \cup y)-\left(p^{i *} x \cup p^{\prime *} y\right),[X]\right\rangle=-\langle x \cup T y,[\bar{X}]\rangle$

$$
\mu: G \rightarrow A ; \quad z \mapsto\left\{\begin{array}{l}
\frac{1}{2} \lambda(z, z) \\
\left\langle S q_{h}^{i+1}\left(\Sigma^{k}\right), \Sigma^{k}[X]\right\rangle
\end{array}\right.
$$

$\left(h=\left(\Sigma^{k} z\right) P-\Sigma^{k}\left(p^{l *} z\right) \in\left[\Sigma^{k} X_{+}, \Sigma^{k} K\left(\mathbb{Z}_{2}, i\right)\right], P: \Sigma^{k} X_{+} \rightarrow \Sigma^{k} \bar{X}_{+}, l \in H^{k}\left(K\left(\mathbb{Z}_{2} ; i\right), \mathbb{Z}_{2}\right)\right.$, $T=$ covering translation $: \bar{X} \rightarrow \bar{X}$ ),

which was used by Browder and Livesay [3] to define a desuspension invariant for fixed point free involutions on spheres, and which more recently has been studied by Brumfiel and Milgram [4]. In general, $\sigma_{*}(p \cup 1, b \cup 1) \neq \sigma_{*}(X, p)$, as has already been shown in the Arf invariant case in Proposition 5.3.1 of [4]. The Poincare transversality obstruction for a double cover $p: \bar{X} \rightarrow X$ of a (4k+2)-dimensional geometric Poincaré complex $X$ obtained by Hambleton and Milgram [8] is the Arf invariant given by the image of $\sigma_{*}(X, p)$ in $L_{4 k+2}(1)=\mathbb{Z}_{2}$.

\section{References}

1. W. Browder, Surgery on simply-connected manifolds (Springer, Berlin 1972).

2. W. Browder, "Cobordism invariants, the Kevaire invariant and fixed point free involutions", Trans. Amer. Math. Soc., 178 (1973), 193-225.

3. W. Browder and G. R. Livesay, "Fixed point free involutions on homotopy sphers", Tohoku Math. J., 25 (1973), 69-88.

4. G. W. Brumfiel and R. J. Milgram, "Normal maps, covering spaces and quadratic functions", Duke Math. $J ., 44(1977), 663-694$.

5. R. Lee, "Semicharacteristic classes", Topology, 12 (1973), 183-199.

6. A. A. Ranicki, "The algebraic theory of surgery I, II", Proc. London Math. Soc., (3), 40 (1980), 87-192 and 193-283.

7. C. T. C. Wall, Surgery on compact manifolds (Academic Press, London and New York, (1970).

8. I. Hambleton and R. J. Milgram, "Poincaré transversality for double covers, Canad. J. Math., 30 (1978), 1319-1330.

Department of Mathematics, Princeton University, Princeton, NJ 08544, U.S.A. 